\title{
A Web Based Multi-Modal Interface for Elderly Users of the Robot-Era Multi-Robot Services
}

\author{
Alessandro Di Nuovo, Frank Broz \\ Tony Belpaeme, Angelo Cangelosi \\ Centre for Robotics and Neural Systems \\ Plymouth University, Plymouth, United Kingdom \\ Filippo Cavallo, Raffaele Esposito \\ Paolo Dario \\ BioRobotics Institute \\ Scuola Superiore Sant'Anna (SSSA), Pisa, Italy \\ \{alessandro.dinuovo,frank.broz,tony.belpaeme,a.cangelosi\}@plymouth.ac.uk $\quad$ f.cavallo,r.esposito,paolo.dario\}@sssup.it
}

\begin{abstract}
In this paper we present the design and technical implementation of a web based Multi-Modal User Interface (MMUI) tailored for elderly users of the robotic services developed by the EU FP7 Large-Scale Integration Project Robot-Era. The project partners are working to significantly enhance the performance and acceptability of technological services for ageing well by delivering a fully realized system based on the cooperation of multiple heterogeneous robots and with the support of an Ambient Assisted Living environment. To this end, elderly users were involved in the definition of the services and in the design of the hardware and software of the robotic platforms from the first stages of the development process and in real experimentation in two test sites. In particular, here we detail the interface software system for multi-modal elderly-robot interaction. The MMUI is designed to run on any device including touch-screen mobiles and tablets that are preferred by the elderly. This is obtained by integrating web based solutions with the Robot-Era middlewares and planner. Finally we present some preliminary results of ongoing experiments to show the successful evaluation of usability by potential users and to discuss the future directions to improve the proposed MMUI software system.
\end{abstract}

\section{INTRODUCTION}

The care of the elderly is one of the great challenges for all the most developed regions (e.g. USA, Europe, Japan, Australia). It is estimated that, by the year 2050, there will be three times more people over the age 85 than there are today and more than $20 \%$ of the population of western countries will be 65 and over [1]. This demographic trend will lead to a growing number of older people living alone and in need of (intensive) care, but, at the same time, more financially well-appointed and wealthy senior citizens ready to enjoy their third age [2]. The rising request for services for ageing well is affecting the growth of the robotics market and research, in which several new platforms were recently presented and tested to improve the independent living of and to take care of the elderly. Indeed, independently living older adults have expressed a willingness to use and accept home robots [3].

In the application domain of elderly care, robotic platforms are usually integrated in an Ambient Assisted Living (AAL) environment [4], in which smart assistance systems and robots are designed and developed for a better and safer life at home, with the objective to enable elderly people to live in their preferred environment longer. In AAL environments many robots may be employed to cover different user needs (e.g. [5]), thus there is the necessity to integrate and enable synergistic cooperation among all these robots that can be deployed to assist the elderly inside or outside their home.

A current challenge for AAL systems is to integrate many ICT services and robotic platforms provided from different manufacturers, therefore a single flexible interface must be designed for such a heterogeneous system [6]. Thus a welldesigned MMUI is one of the most important parts of future robotic systems, is the link between user and the assistive robotic system and it provides the user the indispensable awareness of the system functionalities and the feedback needed to perceive the benefits and make the whole system usable and acceptable.

When applied to AAL, interfaces for robotic platforms share the same design principles and guidelines that are derived from HCI [7]. General principles and examples of recent MMUI for robotics in Ambient Assisted Living Environments are presented and discussed in [8], that present five experimental projects focused on a single platform that was operating inside the user's home. The majority of them use a wheel drive platform with a fixed touch screen as the main Graphic User Interface (GUI). The limitation of the fixed touch screen is that a short distance is needed to interact with the robot. Speech and gesture interfaces may help the human-robot interaction when a GUI is not available, but there are services that require the use of a graphic interface (e.g. shopping, video calling, food ordering, etc...). Multi-Modal User Interfaces (MMUI) target a more human way of interacting with computers, by means of speech, gestures or other modalities, thus are preferred over unimodal interfaces by elderly users [9].

In this scenario, the EU FP7 project Robot-Era [10] aims to innovate the integration of robotics and the AAL paradigm, in order to develop, implement and demonstrate the general feasibility, scientific/technical effectiveness and social/legal plausibility and acceptability by end-users of a plurality of complete advanced robotic services. Integrated services will actively work in real conditions and cooperate with real people to provide favourable independent living, improving the quality of life and the efficiency of care for elderly people. One of the main challenge of the research in the project is to develop services that make use of three different robotic platforms that will be adapted and optimized for the Robot- 
Era services and for usability and acceptability by elderly people in the home (domestic), communal shared living areas (condominium), and outdoor environments.

Within the framework of the Robot-Era project, the commitment of the authors of this contribution is to develop a more inclusive and usable user interface to facilitate interaction with the robots and to operate the novel service system. This work is being done in collaboration with all the project partners to cover all the cross-disciplinary issues. The core of the proposed MMUI is the newest web technology (HTML5, including Javascript, and PHP) that allows for support different interaction modalities from any device connected to the network. In particular the domestic and condominium platforms are equipped with a detachable tablet because it has been found that elderly users consider touch and gesture recognition interfaces easier to use than other forms of interaction (e.g. classic keyboards and mouse) as shown in [11]. In the scientific literature can be found many contributions that specifically provide guidelines and design recommendations about web and tablet interfaces for the elderly. Web design guidelines to address the web experiences of older users can be found in [12], [13], while a survey on touch-based mobile phone interface for the elderly can be found in [14].

The rest of the paper describes the effort done to build such an interface and is organized as follows. Section II briefly describes the user requirements and the Robot-Era platforms and services. Section III details the implementation of the first prototype of MMUI and the software system. Section IV reports preliminary results from the ongoing extensive experimentation phase. Finally section V summarizes this article giving our conclusion and directions of future work to further improve the proposed MMUI.

\section{ELDERLy USER REQUIREMENTS FOR THE ROBOT-ERA PLATFORMS AND THEIR SERVICES}

In order to identify potential robotic services that could improve quality of life, the Robot-Era project involved the elderly in decision-making from the first stages of the design process. A series of focus groups and workshops were conducted with elderly people and stakeholders in the healthcare industry at sites in both Germany and Italy [15]. In particular, four group interviews were conducted in Italy with 40 older people and two in Germany, involving 42 older people. Based on stated needs and desires and on feedback from the technical partners about feasibility, the following set of services were selected for design and implementation during the four years of the project (willingness to use the robot is in parenthesis): transporting/manipulating objects at home (82\%); cleaning (80\%); garbage collection (75\%); surveillance $(73 \%)$; outdoor walking support, indoor escort at night and reminding events, tasks (72\%); laundry support (70\%); for communicating with important persons (68\%); food order and delivery (67\%); shopping/drug order and delivery (62\%).

In the Robot-Era project, these services are being developed to be provided using multiple robots that are each designed to be suited to a particular environment (domestic, outdoor, and

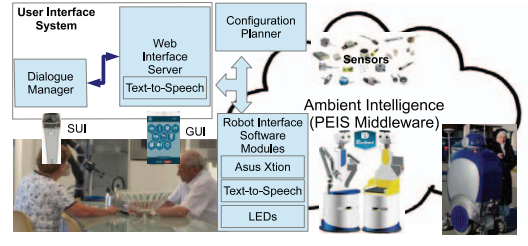

Fig. 1. The Robot-Era MMUI software system and the three platforms.

shared indoor spaces) with the help of an ambient intelligence system. The services are designed and evaluated in two living labs that realistically emulate a home environment.

Regarding the interfaces, the participants reported to clearly prefer a menu with a higher number of buttons, with larger images and icons and without a grouping of functionalities into separate pages that the user must navigate to access services. Also, they preferred speech command/vocal reply for interacting with the robots, that should have a female voice. Other minor suggestions were retrieved from preliminary testing to improve the design, e.g. to add a specific button to go back to the main page because the usual logo icon link was not recognized by the elderly.

\section{Multi-Modal Interface Design for Elderly USERS OF ROBOT-ERA SYSTEM}

In this section we describe in detail the design and implementation of the MMUI for the multi-robot services of Robot-Era. The Robot-Era system is composed of several software modules, that are interconnected using the PEIS Middleware [16] in order to collect information from the environment (i.e. sensors of the apartment and robots). The main modules are shown in Figure 1. Two main modules implement the MMUI: the Web Interface Server that includes the graphic user interface (GUI) and the text-to-speech (TTS) software; the Dialog Manager that implements the Speech User Interface (SUI) with the Automatic Speech Recognition (ASR) software. The current MMUI system offers two main modalities to send commands: they are a GUI, usually running on the tablet attached to the robot, and a SUI, with a wearable microphone on the user. The tablet is mounted on a magnetic frame that can be easily detached. Feedback to the user is given with the same modalities, both graphic and voice (from robot or electronic device), and also using an array of LEDs placed into the domestic robot eyes (e.g. they change colour when the robot is calculating the path). The Configuration Planner Module (CPM), that is responsible for the planning of robot activities and for high-level reasoning, links the MMUI with other devices and modules of the Ambient Intelligence. Details on CPM can be found in [17].

The Robotic platforms are two customized Metralabs SCITOS G5 platforms (Domestic and Condominium), and one RoboTech Dustcart (Outdoor). Each robot runs ROS [18] and MIRA [19] middlewares, the first is used for connecting the additional devices mounted on the robots (e.g. Asus Xtion, LEDs), while the second supports the CogniDrive navigation software that moves the robots. The appearance of the 


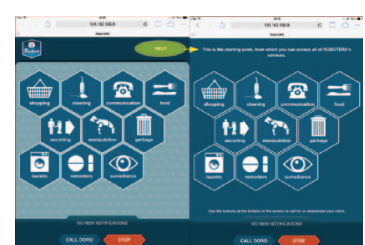

(a)

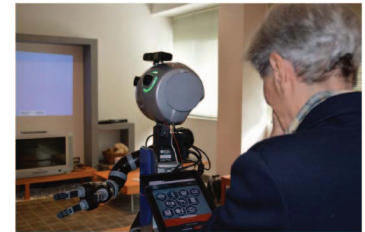

(b)
Fig. 2. (a) GUI main page on the left with HELP button highlighted. On the right the appearance when HELP is active. (b) User interacting with the Domestic Robot during a simulated problem. In case of problems the robot goes to the user and a flashing warning message appears in the middle of the screen, while the alarm sound is played by the tablet.

platforms was also studied with the elderly and specifically designed for the project [20].

\section{A. Web GUI design and implementation}

The web server module implements the Graphical User Interface (GUI). It uses web technology to support the widest range of devices that can be connected to the system network. A main menu index page allows the user to navigate between the different service pages that compose the GUI. There are additional web pages, not accessible by the user, that are available from the web server to allow testing and to support other actors of the Robot-Era system (grocer, caregiver, etc...). A settings page, that is not accessible from the main page, is also present to modify the language, items in the shopping page and to switch test site apartment map. Pages are implemented with HTML5 and Javascript so that they can ideally be available on any browser running on any device. On the web server the software interface with the PEIS middleware is achieved through JSON calls to PHP scripts that do the reasoning and communicate via text file with the PEIS interface module, which runs on the server. The PEIS interface module is programmed in $\mathrm{C}++$. TTS module is implemented as ROS module on robots.

1) Design and implementation Remarks: The graphic interface for the Robot-Era services is developed as a web based server-client architecture in order to allow remote control through mobile devices like tablets and mobile phones. Information from the robot or the ambient environment is also made available to the user via notifications and warnings. The interface is complementary to speech control of the robot. The two modalities are usually interchangeable, except for shopping and communication services where tablet is required for the shopping list and the video call. For video calling, Skype was integrated with the interface using its web API. The graphics interface is intended to run in any platform (e.g. PC, tablet, smartphones) with any web browser, but at the same time the design of the graphic interface aims to maximize the integration with the host device, in order to give the impression that it can be a real product and, moreover, to provide people that have previous knowledge of the device with the basic commands that they already know.

2) Main Page and common interfaces: User can access to services from buttons on the main page (Figure 2(a) on the left) that presents all Robot-Era services. Service interaction is contained in one page only, in order to avoid confusing the user going back and forth through the pages to search for functionalities [21]. To access the desired service the user can click or tap with his finger an hexagon that represents the service you want to access, thus the corresponding page will be shown. As in all other pages, if the user taps/clicks on the HELP, button some text will appear to give basic info about the page. Some common functions (Help, Go back to main MENU, CALL and STOP the DOmestic RObot) are always accessible from the same button on all pages.

3) Graphic Design: The graphic design of the user interface is heavily inspired by the logo / header graphic of Robot-Era, predominantly to keep in line with the branding. Based on the convention whereby interacting with the logo on a website transports the user to the landing page, we derived that every other element that is click-able within the application should have a similar shape. In order to avoid ending up with an interface that feels too modern and robotic, little decorations have been added such as the overall background pattern (a robotic equivalent of a wallpaper). The script font inside the logo introduces certain friendliness, which for the remaining elements are replicated through visuals rather than type in order to maintain legibility. High priority elements such as buttons have their label capitalized in order to draw attention as well as be highly legible. In the two cases in which the screen significantly changes abruptly, the interaction confirmation view and the help view on every page, a solid colour replaces the background in order to single out the respective interface elements. Access to services is possible using only one page in order to avoid confusing the user going back and forth through the pages to search for functionalities.

The icons are meant to be bold and simple. Where possible, we have tried to use as retro a version of a technology so that they are easily recognisable by drawing on long engrained ideas of the elderly as to how things should look, i.e. a phone is recognizable as such, including its old-school rotary dial, so does the vacuum cleaner still have a bag.

\section{B. Speech Interaction and Multi-language support}

A simple dialogue manager is implemented to allow the user to order the robotic services by using spoken commands for the speech user interface (SUI). Speech recognition is implemented using the Nuance SDK and is based on a set of restricted grammars. Nuance was preferred because it supports all the languages used in our experimentation (Italian, German and Swedish, but also English for debugging and demonstration). The program runs as a single-threaded application whose flow is controlled by the audio input stream. Audio events trigger callbacks to handle the processing of sound input. The recognition grammars are loaded dynamically to change what input the system is "listening for" based on the context and stage of the verbal interaction.

The user begins a verbal interaction with the robot by calling the robot by name using their wearable microphone. The robot's name is defined as a "wake-up word" which 
must be recognised before a service request interaction is initiated by the speech interface. This prevents service requests from being issued based on false positives from the speech recognition (which could otherwise occur in situations where the user is speaking to another person present rather than the robot). The keywords used to identify each service are specified in the grammars and may be uttered alone or as part of a longer natural language phrase. During a service request interaction, the user may request any service. The following interaction will be determined by which service was selected. After the user has called the robot, the dialogue proceeds in a system-initiative manner. The speech interface is designed to produce short, simple, command-oriented dialogues with the user. In the case of services which require complex or extended user input, (such as creating a shopping list or entering an appointment for a reminder), the SUI directs the user to use the GUI for input and hands the interaction over to the GUI. This design choice was made to avoid the need for numerous confirmation or error-recovery dialogues which could frustrate the user in the event of low speech recognition accuracy.

The GUI and TTS also support the four languages used for debugging and testing: English, Italian, Swedish and German. Both modules shares the same dictionary that associates a set of keywords with text in all languages supported. The Acapela Voice As A Service(VAAS) is used for TTS. This is a web service that receives any text with voice parameters and responds with an audio file containing the speech. The Acapela web service is a module integrated into the web interface and on the robot platforms. Acapela web VAAS was preferred because of its easy integration with the web architecture of the interface. Voices were selected based on an informal survey of native speakers among the choices available for each language (many options for English but only a couple for each other language). The domestic robot has a female voice, as requested by the elderly in preliminary study, while condominium and outdoor have male voices to distinguish them and their service roles from the domestic robot.

\section{User feedback}

In order to make the system more intuitive for elderly users particular emphasis was given to the types and quality of feedback that the robot interface provides [22]. The MMUI software is also responsible for giving feedback to the user via the same modalities that he use to command the robot. This was implemented by making both robotic platforms and web graphic interface able to produce sounds, including speech. As visual feedback the tablet can show specific text messages and robots can also change colour of their LEDs.

Notification. When the system has to give feedback to the user, the main instrument is the text notification area (always present in middle-bottom of the screen). To attract the attention of the user a sound is repeated until the notification disappears. During notifications the robot may reach the user and use TTS to verbally communicate the notification text to him.

User confirmation. When the system needs the confirmation from the user to execute an operation or to confirm that it is

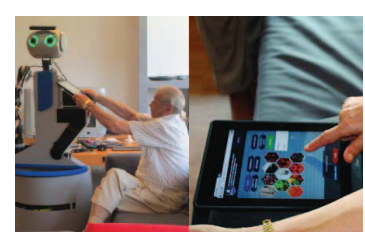

(a)

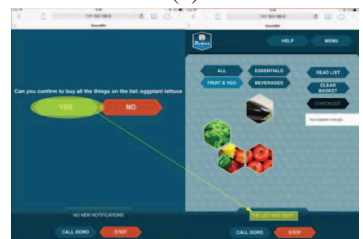

(c)

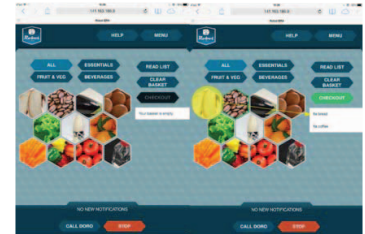

(b)

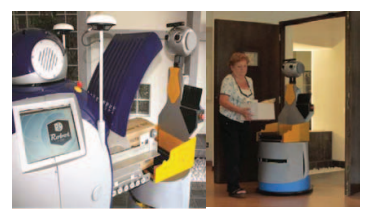

(d)
Fig. 3. (a) When the user calls the domestic robot via SUI, it will ask him to detach the tablet and to use the GUI to create the shopping list. (b) GUI: On the right the list with two items. At this stage it is possible to ask the system use the TTS to read the list aloud by clicking the "READ LIST" button.(c) GUI: The system asks for confirmation and then notifies that the list has been sent. The planner sends the outdoor robot to collect the order from the grocery store.(The grocer uses an additional web page to interact with the outdoor robot.) (d) (left) The system coordinates the exchange between condominium and outdoor platforms. (right) The user is informed by the domestic robot that she can collect the goods from the condominium robot at the entrance door.

done, a message with a confirm button will appear on the page (for an example see the figure 3(c) on the left). The robot may reach the user and verbally communicate the text to confirm.

Warning message. If the ambient intelligence detects a problem, it will be displayed to the user via the activation of a warning message, which flashes in red to attract the user's attention. A specific warning sound will be periodically played and a red bar appears in the middle of the screen as shown in Figure 2(b). In case of warning the CPM commands the robot to reach the user and to notify that there is something wrong (via TTS). Meanwhile a Skype call is automatically started with the caregiver. Warnings cannot be ignored by the user, only the caregiver or ambient intelligence can trigger it off.

Sounds. Sounds will be played for certain events, e.g. text notifications, interactions or warnings, to attract the user attention. The sounds are intended to be pleasant. The notification sound is something user might want to able to notice on a subconscious level, without it getting on his nerves. The sound that signifies an input is needed from the user is slightly longer than the notification sound and the tonal sequence is going in the opposite direction. The warning sound is deep, to invoke a sense of trouble rather than using a siren which means immediate danger.

In picture 3 we briefly present the shopping service as an example of the functionalities offered by the Robot-Era MMUI.

\section{Preliminary RESUlts of EXPERIMENTS AND USABILITY ANALYSIS}

In this section we present some preliminary results of the experimental cycle, that is running in the two test sites of the project (Peccioli in Italy and Örebro in Sweden), which 
are two "living labs" that realistically emulate a home environment. So far, more than 60 elderly are being involved in testing the services of the Robot-Era project. The targeted user is: over 65 years old; with mild or moderate health problems, motor and cognitive deficits; Living alone or with their relatives but without a full-time caregiver. These requirements are motivated by the interest in investigating the interaction between technology and older people and its integration in the daily living of the end users, in order to support and not substitute the relationship between elderly and their family/caregivers.

After the first contact with potential subjects, a recruitment interview was conducted, and, if the subject was be considered suitable, he/she was enrolled. Then, subjects went to one of the testing sites, in which they experienced the Robot-Era services and interacted with the robots by means of use cases. At the end of the testing of each service, subjects completed the final questionnaires. Attitude, usability, acceptance and quality of life are the metrics that constitute the kernel of each protocol, and they were evaluated all along the testing phases by means of qualitative interviews and standardized tools. Full details on the interviews and the experimental protocol for the Robot-Era services and robots can be found on the deliverable D8.1 [23].

Results reported here are from the testing of 7 services, that were experienced by a varying number of subjects: Shopping ( $\mathrm{N}=35)$, Communication ( $\mathrm{N}=53)$, Laundry $(\mathrm{N}=18)$, Reminding $(\mathrm{N}=27)$, Garbage collection ( $\mathrm{N}=35)$, Food delivery $(\mathrm{N}=19)$ and Indoor Escort $(\mathrm{N}=23)$. Shopping, Reminding, Garbage and Indoor escort were tested in the Italian test site (with text and speech in Italian), while Laundry and Food delivery were tested in Sweden (with text and speech in Swedish). Here we have results from both test sites only for the communication service. Use cases were scripted to allow the user to experience all the functionalities of the interface. More than one use case was conceived. For example to test the communication in one case the user starts a Skype call (using the SUI or the GUI). In another case a gas leak is simulated so the warning procedure informs the user and he must answer to a Skype call from the caregiver. In the Indoor Escort at night the user commands the robot using physical buttons attached to the support on the back of the robot, because of this the GUI is not evaluated for this service. Technological background of Italian subjects is that they know little or nothing about recent devices: less than $30 \%$ of them actually use a smart phone or tablet. Conversely, Swedish subjects that use these devices are around $50 \%$.

Subjects did not receive any preliminary explanation on how to use the interface. They were asked to follow first a use case, different for each service, then they had time for free interaction and additional personal testing. To evaluate usability of the interface the following questions were added to the qualitative interview:

1) I feel the robot understood what I wanted to do

2) I could clearly hear what the robot said to me.

3) I found the tablet easy to use to perform the service

4) I could clearly read the messages on the tablet.

5) I understood what buttons I needed to press to perform the service

6) I found it easy to speak to the robot to perform the service

7) I understood what I could say to the robot to perform the service
Subjects were asked to answer on a five-point Likert scale: strong disagree, disagree, no opinion, agree, strongly agree. Average scores and standard deviations per question are summarized in Table I.

From ad-hoc acceptability interviews, the majority of the users evaluated positively the overall experience for all services considered. In particular Italians evaluated "excellent" four of them: Shopping, Garbage, Indoor and Communication. The acceptance ad-hoc questionnaire consists of several items and the users can reply to these statements on a 5 points Likert type scale.

From observation during the experiments and recorded videos, we saw that the subjects, which are not used to technology have initial difficulties using the tablet, they often failed the first attempt and sometimes they needed the help from the interviewer to go on with the service. For the SUI, some subjects have difficulties because they did not say the right keyword to activate the system. Initial problems were mainly due to the lack of training, because usually after a short adaptation time subjects were able to use the interface independently with good success. Indeed, in the final evaluation, they gave good scores to the interface usability as reported in Table I.

We performed a t-test to compare SUI and GUI average scores per subject. A statistically different $(p<0.05)$ result was found for Shopping and Reminding. This due to the fact that there is a difference also in the functionalities offered by the SUI and GUI for both services. Indeed, since we decided to develop a simple SUI, the most complex interaction is carried out via GUI only. For the shopping service the user can compile and send the shopping list only with the tablet. For reminding the user must fill the complete reminding form, while he can use the SUI only to set a simple reminder (e.g. "remind me of something in 5 minutes").

\section{CONCLUSION AND FUtURE WORK}

In this paper we presented the design and technical implementation of a web based MMUI tailored for elderly users of the robotic services developed by the Robot-Era project. Robot-Era objective is to significantly enhance the performance and acceptability of technological services for ageing well by delivering a fully realized system based on the cooperation of multiple heterogeneous robots and with the support of an AAL infrastructure. To this end, preliminary studies with the potential users set the requirements also for the interface. Interaction with the user and feedback are implemented by means of several communication channels (e.g. touch screen, speech, LEDs), that help to achieve a more intuitive interaction and to increase the robustness of the interaction by using redundant or complementary information. This interface can be detached from the single robotic platform and assists the user to easily interoperate with the whole system. Preliminary results of the experimentation in the two test sites shows that the subjects evaluated positively both the graphic and speech interface. This result is mainly due to the 
TABLE I

USABILITY ASSESSMENT

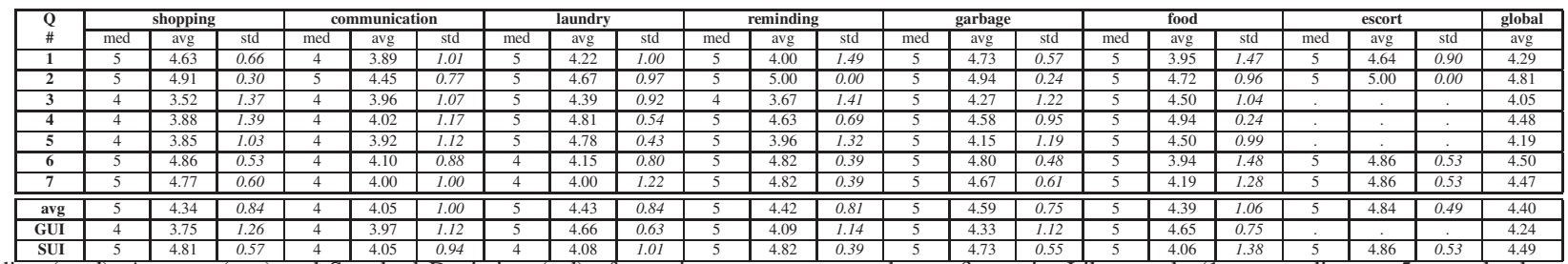

Median (med), Average (avg) and Standard Deviation (std) of question scores expressed on a five point Likert scale (1=strong disagree; $5=$ completely agree) GUI $=$ avg of question 3,4 and 5; SUI $=$ avg of question 6 and 7. The difference is statistically significant $(p<0.05)$ only for Shopping and Reminding.

simplicity of the design that allows also inexperienced users to successfully interact the system.

The project will run until the end of 2015 , so we aim to further improve the acceptability and usability and to extend the functionalities of the interface by introducing intelligent behaviours. Future work will focus on human-robot interaction by increasing the feedback to the user using the full range of modalities available to the system through both the GUI and the robot's actions (e.g. accompanying speech with nonverbal behaviours such as gesture and gaze).

\section{ACKNOWLEDGMENT}

The research leading to these results has received funding from the European Union Seventh Framework Programme (FP7/2007-2013) under grant agreement n. 288899.

\section{REFERENCES}

[1] T. Plowman, D. Prendergast, S. Roberts, and J. Sherry, "The global aging experience project," Ethnographic Research, Intel Research, 2010.

[2] G. Van Den Broek, F. Cavallo, and C. Wehrmann, AALIANCE Ambient Assisted Living Roadmap. Amsterdam, The Netherlands, The Netherlands: IOS Press, 2010

[3] C.-A. Smarr, A. Prakash, J. M. Beer, T. L. Mitzner, C. C. Kemp, and W. A. Rogers, "Older adults preferences for and acceptance of robot assistance for everyday living tasks," in Proceedings of the Human Factors and Ergonomics Society Annual Meeting, vol. 56, no. 1. SAGE Publications, 2012, pp. 153-157.

[4] F. Cavallo, M. Aquilano, M. Bonaccorsi, R. Limosani, A. Manzi, M. C. Carrozza, and P. Dario, "On the design, development and experimentation of the astro assistive robot integrated in smart environments," in Robotics and Automation (ICRA), 2013 IEEE International Conference on. IEEE, 2013, pp. 4310-4315.

[5] R. Li, M. A. Oskoei, and H. Hu, "Towards ros based multi-robot architecture for ambient assisted living," in Systems, Man, and Cybernetics (SMC), 2013 IEEE International Conference on. IEEE, 2013, pp. 34583463.

[6] T. Kleinberger, M. Becker, E. Ras, A. Holzinger, and P. Müller, "Ambient intelligence in assisted living: enable elderly people to handle future interfaces," in Universal access in human-computer interaction. Ambient interaction. Springer, 2007, pp. 103-112.

[7] M. Petzold, F. Barbabella, J. Bobeth, D. Kern, C. Mayer, and M. Morandell, "Towards an ambient assisted living user interaction taxonomy," in CHI'13 Extended Abstracts on Human Factors in Computing Systems. ACM, 2013, pp. 49-54.

[8] P. Mayer, C. Beck, and P. Panek, "Examples of multimodal user interfaces for socially assistive robots in ambient assisted living environments," in Cognitive Infocommunications (CogInfoCom), 2012 IEEE 3rd International Conference on. IEEE, 2012, pp. 401-406.

[9] C. Jian, H. Shi, F. Schafmeister, C. Rachuy, N. Sasse, H. Schmidt, V. Hoemberg, and N. Steinbchel, "Touch and speech: Multimodal interaction for elderly persons," in Biomedical Engineering Systems and Technologies, ser. Communications in Computer and Information Science, J. Gabriel, J. Schier, S. Huffel, E. Conchon, C. Correia, A. Fred, and H. Gamboa, Eds. Springer Berlin Heidelberg, 2013, vol. 357, pp. $385-400$.
[10] "Robot-Era project: Implementation and integration of advanced robotic systems and intelligent environments in real scenarios for the ageing population," FP7 - ICT - Challenge 5: ICT for Health, Ageing Well, Inclusion and Governance. Grant agreement number 288899. [Online]. Available: www.robot-era.eu

[11] B. Loureiro and R. Rodrigues, "Multi-touch as a natural user interface for elders: A survey," in Information Systems and Technologies (CISTI), 2011 6th Iberian Conference on. IEEE, 2011, pp. 1-6.

[12] P. Zaphiris, S. Kurniawan, and M. Ghiawadwala, "A systematic approach to the development of research-based web design guidelines for older people," Universal Access in the Information Society, vol. 6, no. 1, pp. 135-136, 2007.

[13] A. Arch, S. Abou-Zahra, and S. L. Henry, "Older users online: WAI guidelines address the web experiences of older users," User Experience Magazine, vol. 8, no. 1, pp. 18-19, 29, 2009.

[14] M. Al-Razgan, H. Al-Khalifa, M. Al-Shahrani, and H. AlAjmi, "Touchbased mobile phone interface guidelines and design recommendations for elderly people: A survey of the literature," in Neural Information Processing, ser. Lecture Notes in Computer Science, T. Huang, Z. Zeng, C. Li, and C. Leung, Eds. Springer Berlin Heidelberg, 2012, vol. 7666, pp. $568-574$

[15] R. Bevilacqua, E. Felici, F. Marcellini, S. Klemcke, C. Nedopil S. Glende, F. Cavallo, M. Aquilano, M. Carrozza, and P. Dario, "Robotera project (fp7-ict-2011.5.4): from the end-users perspective to robotics. preliminary findings." in Proceedings of the AAL - Ambient Assisted Living Forum, 2012.

[16] A. Saffiotti, M. Broxvall, M. Gritti, K. LeBlanc, R. Lundh, J. Rashid, B. Seo, and Y.-J. Cho, "The peis-ecology project: vision and results," in IEEE/RSJ International Conference on Intelligent Robots and Systems (IROS). IEEE, 2008, pp. 2329-2335.

[17] M. Di Rocco, S. Sathyakeerthy, J. Grosinger, F. Pecora, A. Saffiotti, M. Bonaccorsi, F. Cavallo, R. Limosani, A. Manzi, G. Teti, and D. Paolo, "A planner for ambient assisted living: From high-level reasoning to low-level robot execution and back," in 2014 AAAI Spring Symposium Series, 2014.

[18] M. Quigley, K. Conley, B. Gerkey, J. Faust, T. Foote, J. Leibs, R. Wheeler, and A. Y. Ng, "Ros: an open-source robot operating system," in ICRA workshop on open source software, vol. 3, no. 3.2, 2009.

[19] E. Einhorn, T. Langner, R. Stricker, C. Martin, and H. Gross, "Mira middleware for robotic applications," in Intelligent Robots and Systems (IROS), 2012 IEEE/RSJ International Conference on, Oct 2012, pp. 2591-2598.

[20] N. Casiddu, F. Cavallo, A. Divano, I. Mannari, E. Micheli, C. Porfirione, M. Zallio, M. Aquilano, and P. Dario, "Robot interface design of domestic and condominium robot for ageing population," in ForITAAL, October 2013.

[21] M. Torun, T. van Kasteren, O. D. Incel, and C. Ersoy, "Complexity versus page hierarchy of a gui for elderly homecare applications," in Computers Helping People with Special Needs. Springer, 2012, pp. 689-696.

[22] F. Broz, A. D. Nuovo, T. Belpaeme, and A. Cangelosi, "Multimodal robot feedback for eldercare," in Workshop on Robot Feedback in Human-Robot Interaction: How to Make a Robot Readable for a Human Interaction Partner at Ro-MAN 2012, 2012.

[23] F. Marcellini, R. Bevilacqua, E. Felici, S. Klemcke, S. Glende, R. Esposito, and F. Cavallo, "Experimental protocol for the first cycle of experiments of Robot-Era services," 2013, Robot-Era Project Deliverable D8.1. [Online]. Available: www.robot-era.eu 